\title{
Correlação entre os achados clínicos e imaginológicos nas disfunções temporomandibulares*
}

\author{
Correlation between clinical and imaging findings in patients with temporomandibular disorders
}

Fábio Augusto Cozzolino ${ }^{1}$, Abrão Rapoport ${ }^{2}$, Sérgio Altino Franzi ${ }^{3}$, Ricardo Pires de Souza ${ }^{3}$, Clemente Augusto de Brito Pereira ${ }^{3}$, Rogério Aparecido Dedivitis ${ }^{3}$

Resumo OBJETIVO: Verificar a relação entre sinais e sintomas observados no exame clínico de pacientes com diagnóstico de disfunção temporomandibular, conforme os resultados fornecidos pelo exame de ressonância magnética. MATERIAIS E MÉTODOS: Trinta pacientes que apresentavam sinais e sintomas de disfunção temporomandibular foram submetidos a exame clínico e de ressonância magnética. Cada exame de ressonância magnética de articulação temporomandibular foi interpretado, independentemente, por dois radiologistas experientes. Os exames de ressonância magnética foram realizados com 12 cortes de $3 \mathrm{~mm}$ de espessura, em orientação coronal (T1) em posição de boca fechada, cortes sagitais em posição de boca aberta e fechada (T1 e T2) e em abertura e fechamento progressivos, com intervalo de $5 \mathrm{~mm}$, para reproduzir toda a extensão do movimento mandibular. A significância estatística entre a análise clínica dos pacientes com disfunção temporomandibular e os resultados obtidos no exame de ressonância magnética foi avaliada pelo teste kappa. RESULTADOS: Obteve-se, na análise interobservadores de imagens, concordância bruta do lado esquerdo e direito, respectivamente, de 56,7\% (kappa $=0,1)$ e 56,7 (kappa $=0$ ). CONCLUSÃO: Não foi encontrada correlação entre o diagnóstico clínico da luxação discal e imagens de ressonância magnética. Unitermos: Ressonância magnética; Articulação temporomandibular; Distúrbios.

Abstract OBJECTIVE: To correlate the signals and symptoms observed on clinical examination of patients with temporomandibular disorder with the results demonstrated by magnetic resonance imaging. MATERIALS AND METHODS: Thirty patients presenting with signs and symptoms of temporomandibular disorders underwent clinical evaluation and subsequent magnetic resonance imaging. The magnetic resonance imaging studies were independently evaluated by two experienced radiologists. Magnetic resonance imaging studies consisted of 12 images in coronal, T1-weighted sequences with $3 \mathrm{~mm}$-thick slices with the mouth closed, sagittal, T1- and T2-weighted sequences with both open and closed mouth positions, and on progressive opening/closing movement at $5 \mathrm{~mm}$ intervals, in order to demonstrate the full mandibular movement. The statistical significance between the clinical findings in the evaluation of the patients and results found on the magnetic resonance imaging studies was analyzed by means the kappa test. RESULTS: Interobserver agreement was respectively $56.7 \%(\mathrm{kappa}=0.1)$ and $56.7(\mathrm{kappa}=0)$ for the left and right sides. CONCLUSION: No correlation was found between the clinical and magnetic resonance imaging findings in the diagnoses of disc displacement.

Keywords: Magnetic resonance imaging; Temporomandibular joint; Disorders.

Cozzolino FA, Rapoport A, Franzi SA, Souza RP, Pereira CAB, Dedivitis RA. Correlação entre os achados clínicos e imaginológicos nas disfunções temporomandibulares. Radiol Bras. 2008;41(1):13-17.

\section{INTRODUÇÃO}

Os desarranjos internos da articulação temporomandibular (ATM) determinam quadros dolorosos e disfunções mandibulares, bem como sintomas que acometem primariamente os tecidos moles dessa ar-

* Trabalho realizado no Hospital Heliópolis (Hosphel), São Paulo, SP, Brasil.

1. Mestre em Ciências da Saúde pelo Curso de Pós-graduação do Hospital Heliópolis (Hosphel), São Paulo, SP, Brasil.

2. Livre-Docente, Docente Permanente do Curso de Pós-graduação em Ciências da Saúde do Hospital Heliópolis (Hosphel) São Paulo, SP, Brasil.

3. Doutores, Docentes Permanentes do Curso de Pós-graduação em Ciências da Saúde do Hospital Heliópolis (Hosphel), São Paulo, SP, Brasil. ticulação e o posicionamento do disco articular.

Dessa forma, a imagem traz grande contribuição para o diagnóstico final e avaliação terapêutica. As técnicas radiográficas convencionais (panorâmicas, transcranianas) e especiais para ATM (artrografias, artrotomografias, tomografias convencionais e computadorizadas) possuem limitações devido a localização, composição,

Endereço para correspondência: Dr. Abrão Rapoport. Rua Iramaia, 136, Jardim Europa. São Paulo, SP, Brasil, 01450-020. E-mail: arapoport@terra.com.br

Recebido para publicação em 20/12/2006. Aceito, após revisão, em 4/6/2007. complexidade e tamanho da articulação, além do grau de irradiação ionizante a que o paciente é exposto.

A ressonância magnética $(\mathrm{RM})$ veio revolucionar o diagnóstico e o tratamento das disfunções temporomandibulares (DTMs), por proporcionar imagens multiplanares de alta precisão dos tecidos duros e moles da ATM, sem mudar o paciente de posição e sem a utilização de irradiação ionizante. A RM tem sido o exame de imagem de primeira escolha para o diagnóstico das anormalidades de tecido mole da ATM, pois apresenta alta acurácia na determinação da posição do disco $\operatorname{articular}^{(\mathbf{1})}$. 
Nosso objetivo foi verificar a relação entre sinais e sintomas observados no exame clínico de pacientes com diagnóstico de DTM, conforme os resultados fornecidos pelo exame de RM.

\section{MATERIAIS E MÉTODOS}

Neste estudo retrospectivo foram admitidos 30 pacientes que procuraram o Serviço de Odontologia da Universidade Cruzeiro do Sul, com diagnóstico de DTM, no período de janeiro de 2002 a janeiro de 2006. Esse projeto foi aprovado pelo Comitê de Ética em Pesquisa da Universidade Cruzeiro do Sul, sob o protocolo $n^{\circ}$ 036/05. Os critérios de inclusão foram pacientes com DTM com quadro de cefaléia, dor auricular e pré-auricular e orofacial. Os critérios de exclusão foram pacientes tratados previamente por cirurgia e menores de 18 anos de idade.

Cada exame de RM de ATM foi interpretado, independentemente, por dois radiologistas experientes que, segundo os critérios estabelecidos por Nebbe et al. ${ }^{(2)}$ (Tabela 1; Figuras 1 e 2), formularam laudos de diagnóstico. Quando houve discor-

Tabela 1 Critérios estabelecidos por Nebbe et al. ${ }^{(2)}$.
a. Posição normal do disco articular.
b. Deslocamento anterior leve do disco articular com redução em abertura
c. Deslocamento anterior moderado do disco articular com redução em abertura.
d. Deslocamento anterior total do disco articular com redução em abertura.
e. Deslocamento anterior total do disco articular sem redução em abertura.
f. Outras categorias da posição discal.

dância entre os laudos de diagnóstico, um resultado final foi decidido por consenso entre os radiologistas.

Os indicadores consistiam em um questionário proposto pela Academia Americana de Dor Orofacial - Diretrizes para Classificação, Acesso e Tratamento da Disfunção Temporomandibular ${ }^{(3)}$. A queixa principal foi obtida por meio de entrevista, como parte inicial do exame clínico. A seguir, por meio de palpação bilateral, foi verificada a presença de dor articular, lateral e posterior à cápsula, e realizada a ausculta para verificação de ruídos articulares (estalos e crepitação). A presença de dor muscular foi avaliada por palpação bilateral dos seguintes músculos ou regiões: masseter superficial, masseter profundo, temporal anterior, temporal posterior, re- gião frontal, região de vértex, cervicais posteriores, digástrico e esternocleidomastóideo, por via extrabucal; e inserção do temporal, pterigóideo medial e pterigóideo lateral, por via intrabucal.

Segundo estudos anteriores ${ }^{(4-6)}$, os sons articulares da ATM são: estalo ou estalido - é um breve barulho que ocorre em alguns pontos durante a abertura, fechamento ou lateralidade; e crepitação — é um som de rangido de tal forma que seja perceptível fracamente, sugestivo de esclerose subcondral.

Os exames de RM foram realizados em um aparelho Gyroscan ACS-NT, 1,5 tesla (Philips Co.; Amsterdam, Holanda). Foram realizados 12 cortes de $3 \mathrm{~mm}$ de espessura com bobina de superfície, em orientação coronal, em seqüência T1 (400/20 ms, FOV

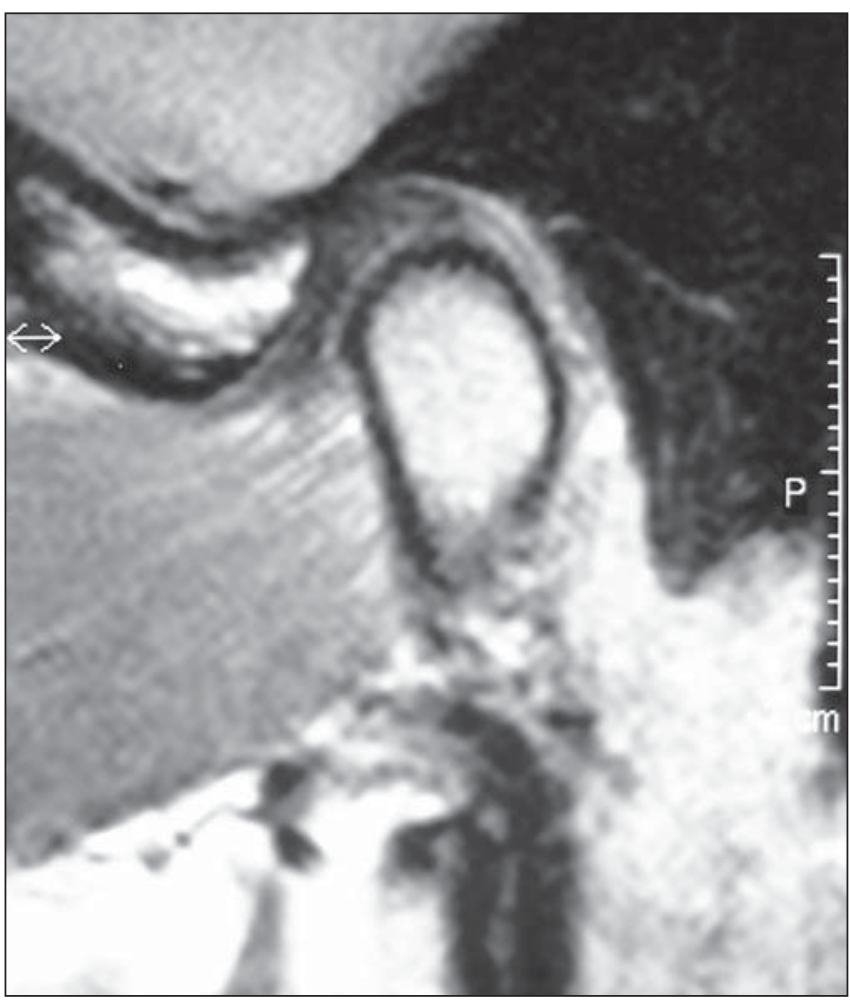

Figura 1. Corte sagital de RM com a boca fechada evidenciando posicionamento normal do disco.

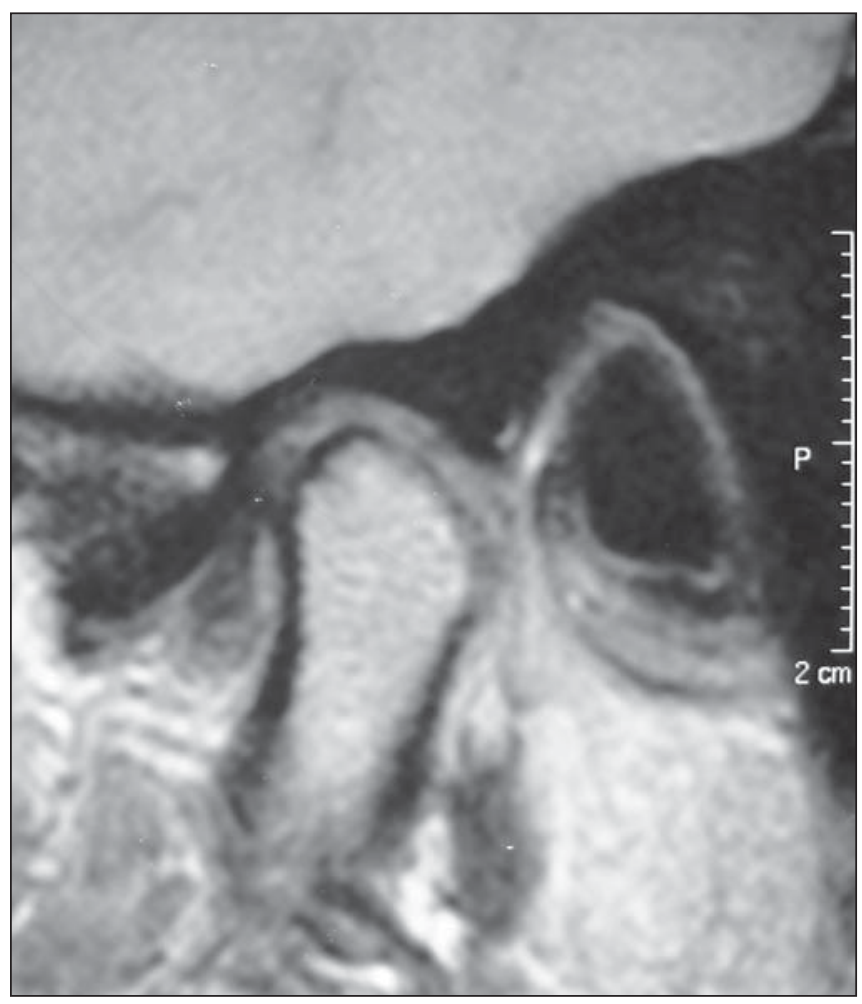

Figura 2. Corte sagital de RM com a boca fechada evidenciando deslocamento anterior do disco. 
= $19 \mathrm{~cm}$ ), em posição de boca fechada, cortes sagitais em posição de boca aberta e fechada, em seqüências T1 (400/20 ms, $\mathrm{FOV}=19 \mathrm{~cm})$ e T2 $(2.670 / 15 \mathrm{~ms}, \mathrm{FOV}=$ $19 \mathrm{~cm})$, e em abertura e fechamento progressivos (três posições - aberturas máxima e intermediária e boca fechada), com intervalo de $5 \mathrm{~mm}$ e flip de $30^{\circ}$ para que se pudesse utilizar o efeito "cine", na tentativa de reproduzir toda a extensão do movimento mandibular e proporcionar imagens dinâmicas gravadas em vídeo. Todas as imagens foram obtidas com o paciente em decúbito dorsal.

Foi realizada estatística descritiva dos dados obtidos com relação a gênero e faixa etária, dor facial, sons articulares e posição do disco articular na RM.

Com o objetivo de estabelecer a relação entre a RM, a dor facial e os sons articulares, foram realizadas as interações segundo o diagnóstico clínico versus $\mathrm{RM}$, dos lados direito e esquerdo, respectivamente. A significância estatística entre a análise clínica dos pacientes com DTM e os resultados obtidos no exame de RM foi avaliada por meio do teste kappa, que determinou o nível de correlação.

\section{RESULTADOS}

Observou-se predominância do gênero feminino (24 pacientes), com proporção de 3,2:1 em relação ao masculino, e maior ocorrência na faixa etária de 18 a 29 anos, com $41,7 \%$ do total de pacientes. Quanto ao quadro doloroso, $14(46,7 \%)$ pacientes apresentavam dor bilateral, 4 (13,3\%), dor à direita, $3(10,0 \%)$, dor à esquerda e 9 $(30,0 \%)$ não referiam dor.

Quanto à presença ou ausência de ruídos articulares nos pacientes, o lado direito foi acometido em 7 (23,3\%), o esquerdo em $7(23,3 \%)$, bilateralmente em $6(20,0 \%)$ e ausência em 10 (33,3\%). Para os diferentes sons articulares, no lado esquerdo houve estalo em $12(40,0 \%)$ pacientes, crepitação em $1(3,3 \%)$ e ausência em $17(56,7 \%)$. No lado direito, estalo foi diagnosticado em 12 $(40,0 \%)$ pacientes, crepitação em $1(3,3 \%)$ e ausência em 17 (56,7\%).

A Tabela 2 mostra a freqüência de alterações articulares e a distribuição com relação ao lado do paciente, de acordo com os laudos obtidos nas RMs.
As Tabelas 3 e 4 apresentam a freqüência das alterações articulares e a incidência de cada tipo de alteração, de acordo com os laudos das RMs nos lados esquerdo e direito.

A Tabela 5 apresenta a relação entre o diagnóstico clínico de luxação do lado esquerdo e o diagnóstico do exame de RM no lado esquerdo, enquanto os achados à direita são apresentados na Tabela 6 .

\section{DISCUSSÃO}

Procuramos conjugar o uso da RM, que, como já foi dito, é o exame de imagem mais indicado para a avaliação da ATM e seus

Tabela 2 Distribuição da freqüência de alterações articulares e a distribuição com relação ao lado do paciente.

\begin{tabular}{|lcc|}
\hline Variável & Freqüência & Porcentagem \\
\hline Normal & 9 & $30,0 \%$ \\
RM do lado direito & 3 & $10,0 \%$ \\
RM do lado esquerdo & 5 & $16,7 \%$ \\
Ambos & 13 & $43,3 \%$ \\
Total & 30 & $100,0 \%$ \\
\hline
\end{tabular}

Tabela 3 Distribuição dos resultados do exame de RM do lado esquerdo.

\begin{tabular}{|lcc|}
\hline Variável & Freqüência & Porcentagem \\
\hline Normal & 14 & $46,7 \%$ \\
Luxação anterior com redução leve & 9 & $10,0 \%$ \\
Luxação anterior com redução moderada & 2 & $6,7 \%$ \\
Luxação anterior com redução severa & 3 & $10,0 \%$ \\
Luxação anterior sem redução & 2 & $6,7 \%$ \\
Total & 30 & $100,0 \%$ \\
\hline
\end{tabular}

Tabela 4 Distribuição dos resultados do exame de RM do lado direito.

\begin{tabular}{|lrc|}
\hline Variável & Freqüência & Porcentagem \\
\hline Normal & 12 & $40,0 \%$ \\
Luxação anterior com redução leve & 10 & $33,3 \%$ \\
Luxação anterior com redução moderada & 1 & $3,3 \%$ \\
Luxação anterior com redução severa & 4 & $13,3 \%$ \\
Luxação anterior sem redução & 3 & $10,0 \%$ \\
Total & 30 & $100,0 \%$ \\
\hline
\end{tabular}

Tabela 5 Distribuição do diagnóstico de luxação pela RM no lado esquerdo segundo o diagnóstico clínico de luxação.

\begin{tabular}{|llccc|}
\hline \multirow{2}{*}{ Variáveis } & \multicolumn{2}{c|}{ Diagnóstico clínico de luxação (lado esquerdo) } \\
\cline { 3 - 5 } & & Normal & Com luxação & Total \\
\hline \multirow{2}{*}{ RM } & Normal & 4 & 10 & 14 \\
& Luxação RM & $28,6 \%$ & $71,4 \%$ & $100,0 \%$ \\
& Luxação clínico & $57,1 \%$ & $43,5 \%$ & $46,7 \%$ \\
& Com luxação & 3 & 13 & 16 \\
\multirow{2}{*}{ Total } & Luxação RM & $18,8 \%$ & $81,3 \%$ & $100,0 \%$ \\
& Luxação clínico & $42,9 \%$ & $56,5 \%$ & $53,3 \%$ \\
& Luxação RM & 7 & 23 & 30 \\
& Luxação clínico & $23,3 \%$ & $76,7 \%$ & $100,0 \%$ \\
\end{tabular}

A concordância bruta do lado esquerdo foi de $56,7 \%$ (kappa $=0,1 ; p=0,526$ ). 
Tabela 6 Distribuição do diagnóstico de luxação pela RM no lado direito segundo o diagnóstico clínico de luxação.

\begin{tabular}{|llccc|}
\hline \multirow{2}{*}{ Variáveis } & \multicolumn{3}{c|}{ Diagnóstico clínico de luxação (lado direito) } \\
\cline { 2 - 5 } & Normal & Com luxação & Total \\
\hline \multirow{2}{*}{ RM } & Normal & 3 & 9 & 12 \\
& Luxação RM & $16,7 \%$ & $83,3 \%$ & $100,0 \%$ \\
& Luxação clínico & $40,0 \%$ & $40,0 \%$ & $40,0 \%$ \\
& Com luxação & 3 & 15 & 18 \\
& Luxação RM & $16,7 \%$ & $83,3 \%$ & $100,0 \%$ \\
& Luxação clínico & $60,0 \%$ & $60,0 \%$ & $60,0 \%$ \\
Total & 6 & 24 & 30 \\
& Luxação RM & $20,0 \%$ & $80,0 \%$ & $100,0 \%$ \\
& Luxação clínico & $100,0 \%$ & $100,0 \%$ & $100,0 \%$ \\
\hline
\end{tabular}

A concordância bruta do lado direito foi de $56,7 \%($ kappa $=0 ; p=1)$.

componentes, com o uso de um questionário clínico. A RM é considerada um dos melhores exames para a pesquisa do funcionamento da ATM, pois, além de ser método não-invasivo e sem efeitos colaterais, tem boa fidelidade, chegando a ser comparada às artrografias no que se refere à visão de estruturas em funcionamento. Contudo, a associação do exame clínico com o de imagem é fundamental para um correto diagnóstico e prognóstico das DTMs $^{(4)}$. Os dados coletados por meio da anamnese e do exame clínico dos pacientes são a base para o estabelecimento de um diagnóstico correto das DTMs ${ }^{(7)}$.

Há grande concordância entre os observadores na avaliação da ATM pela RM, o que torna esse exame um instrumento de grande aceitação e credibilidade ${ }^{(\mathbf{8 - 1 2})}$. Muitas vezes, os clínicos não conhecem a verdadeira natureza das DTMs de que tratam, pois somente se baseiam em dados clínicos para o estabelecimento do diagnóstico ${ }^{(13)}$. Todavia, os profissionais devem ter conhecimento da imagem para recomendá-la e interpretá-la.

A predominância do gênero feminino, verificada neste grupo, está consoante ao demonstrado em outros estudos sobre DTMs $^{(\mathbf{1 4})}$, que avaliaram 73 pacientes (56 mulheres e 17 homens). Avaliamos pacientes segundo a distribuição da dor facial e observamos que a dor mais comum ocorria em ambos os lados, com 14 pacientes $(46,7 \%)$, dados concordantes descritos em casuísticas de disfunção de $\operatorname{ATM}^{(\mathbf{1 5})}$. Em estudo sobre as alterações morfológicas do processo estilóide em pacientes com DTM, estas foram encontradas em 74 pacientes do gênero feminino e em 9 do gênero masculino, concentrados na faixa etária dos 41 aos $50 \operatorname{anos}(32,5 \%)^{(\mathbf{1 6})}$.

Realizando uma análise descritiva, verificamos que a presença de estalo foi o achado clínico mais comum - 20 casos $(63,3 \%)$, sendo $14(46,6 \%)$ unilaterais e 6 $(20 \%)$ bilaterais — , enquanto outros ${ }^{(17)}$, estudando 98 pacientes, observaram que $60 \%$ possuíam sons unilaterais e $40 \%$ possuíam sons bilaterais.

Na Tabela 2 verificamos que as alterações articulares ocorrem, em maior número, em ambos os lados, num total de 13, resultado semelhante ao de outro estudo ${ }^{(\mathbf{1 8})}$. Verificamos que 34 articulações (70\%) apresentaram algum tipo de alteração no resultado da RM, sendo que a alteração que mais se apresentou foi a luxação anterior com redução leve, com 19 casos (Tabelas 3 e 4). Em outro estudo ${ }^{(19)}$ observou-se que $37,3 \%$ dos pacientes tiveram diagnóstico de luxação discal leve pela RM, enquanto $74,4 \%$ apresentaram luxação discal severa. Quando fizemos as interações entre o diagnóstico pela RM do lado direito e o diagnóstico clínico de luxação do mesmo lado (Tabelas 5 e 6), observamos que 12 (100\%) pacientes tiveram o diagnóstico do exame de RM normal, e destes, 9 (83,3\%) tiveram o diagnóstico clínico de luxação. Do mesmo lado, 18 (100\%) pacientes tiveram o diagnóstico de luxação pela RM, e destes, 15 $(83,3 \%)$ tiveram diagnóstico clínico de luxação. Obtivemos concordância bruta do lado direito de 56,7\% (kappa $=0 ; p=1)$. Do lado esquerdo, observamos que de 14 (100\%) pacientes que apresentaram RM normal, $10(71,4 \%)$ tiveram o diagnóstico clínico de luxação e que de 16 (100\%) pacientes que tiveram o resultado da RM positivo para luxação, $13(81,3 \%)$ tiveram o diagnóstico clínico de luxação. A concordância bruta do lado esquerdo foi de $56,7 \%$ (kappa $=0,1 ; p=0,526$ ). Resultados semelhantes foram obtidos em 46 pacientes com luxação discal com redução, comparando com o exame clínico, ocorrendo concordância bruta de 40,7 e kappa $=0,2^{(\mathbf{2 0})}$.

Com esses resultados, verificamos que a imagem de RM não significa a presença de sintomas dolorosos e vice-versa.

A dor é algo extremamente individual, cujo limiar pode variar bastante entre os pacientes, e seu tipo e localização podem significar fatores etiológicos distintos. No caso das DTMs, vários fatores contribuem para a dor. Entretanto, foi encontrada alta prevalência de pacientes sem sintomas e com deslocamentos do disco com e sem redução (33\%). Mas os pacientes sintomáticos são mais numerosos e representam até $77 \%$ da ocorrência de deslocamento ${ }^{(\mathbf{2 1 , 2 2})}$.

Em nosso estudo, observamos alta prevalência de pacientes com sintomas para DTM (19 articulações) que não apresentavam alterações na RM. Observando individualmente os exames de RM, pudemos encontrar as seguintes situações: pacientes que apresentavam estalo e que, à RM, mostraram-se com o disco em posição normal, sem deslocamento; e pacientes que apresentaram estalos e que, à RM, mostraramse com o disco em posição de deslocamento anterior sem e com redução. Em outra série, não se detectou correlação entre o grau de deslocamento do disco e a dor à palpação dos músculos mastigatórios, ruídos ou achados oclusais ${ }^{(23)}$, nem relação entre a severidade dos sintomas com o grau de deslocamento do disco ${ }^{(24)}$. Contudo, outra série mostra significante relação entre a imagem de RM e o exame clínico ${ }^{(25)}$. Outro estudo com pacientes e $\mathrm{RM}^{(\mathbf{2 6})}$ correlacionou estalos em 36\% das ATMs com posição normal do disco e em $82 \%$ das ATMs com deslocamento com redução, concluindo-se que o diagnóstico clínico de estalos não pode ser utilizado como regra para a determinação da presença e do tipo de deslocamento existente.

O estalo não deve ser considerado sinal patognomônico desse deslocamento, pois foi observado que é o sinal mais frequiente 
em pacientes com suspeita de deslocamento do disco, mas foi encontrado apenas em $53 \%$ desses pacientes e apenas $7 \%$ de crepitação ${ }^{(27)}$. Em nosso estudo, notamos que os sinais clínicos, estalos ou crepitação não são suficientes para se diagnosticar algum tipo de alteração discal.

\section{REFERÊNCIAS}

1. Ramos ACA, Sarmento VA, Campos PSF, et al. Articulação temporomandibular - aspectos normais e deslocamentos de disco: imagem por ressonância magnética. Radiol Bras. 2004;37:44954.

2. Nebbe B, Brooks SL, Hatcher D, et al. Magnetic resonance imaging of the temporomandibular joint: interobserver agreement in subjective classification of disk status. Oral Surg Oral Med Oral Pathol Oral Radiol Endod. 2000;90:102-7.

3. Dworkin SF, LeResche L. Research diagnostic criteria for temporomandibular disorders: review, criteria, examinations and specifications, critique. J Craniomandib Disord. 1992;6:301-55.

4. Manfredini D, Tognini F, Zampa V, et al. Predictive value of clinical findings for temporomandibular joint effusion. Oral Surg Oral Med Oral Pathol Oral Radiol Endod. 2003;96:521-6.

5. Lobo LFL, Nunes LJ. ATM: diagnóstico e tratamento. São Paulo: Pancast; 2000.

6. Prado SD, Pereira HP, Gonçalves A. Ruídos articulares: métodos de detecção e tratamento. Rev Serv ATM. 2003;3:60-5.

7. Brasileiro CB, Cardoso VN, Ruckert B, et al. Avaliação de processos inflamatórios na articulação temporomandibular empregando leucócitos autólogos marcados com tecnécio-99m em modelo animal. Radiol Bras. 2006;39:283-6.

8. Brandlmaier I, Grüner S, Rudisch A, et al. Validation of the clinical diagnostic criteria for temporomandibular disorders for the diagnostic sub- group of degenerative joint disease. J Oral Rehabil. 2003;30:401-6.

9. Taskaya-Yilmaz N, Ogütcen-Toller M. Clinical correlation of MRI findings of internal derangements of the temporomandibular joints. Br J Oral Maxillofac Surg. 2002;40:317-21.

10. Liedberg J, Panmekiate S, Petersson A, et al. Evidence-based evaluation of three imaging methods for the temporomandibular disc. Dentomaxillofac Radiol. 1996;25:234-41.

11. Raustia AM, Pyhtinen J, Tervonen O. Clinical and MRI findings of the temporomandibular joint in relation to occlusion in young adults. Cranio. 1995;13:99-104.

12. Sano T, Westesson PL. Magnetic resonance imaging of the temporomandibular joint. Increased T2 signal in the retrodiskal tissue of painful joints. Oral Surg Oral Med Oral Pathol Oral Radiol Endod. 1995;79:511-6.

13. Pharoah MJ. The prescription of diagnostic images for temporomandibular joint disorders. J Orofac Pain. 1999;13:251-4.

14. Taskaya-Yilmaz N, Ogütcen-Toller M. Magnetic resonance imaging evaluation of temporomandibular joint disc deformities in relation to type of disc displacement. J Oral Maxillofac Surg. 2001;59:860-6.

15. Tanaka EE. Análise dos efeitos da terapia com placas estabilizadoras em pacientes com disfunções têmporo-mandibulares por meio da ressonância magnética. (Tese de Doutorado). São Paulo: Universidade de São Paulo; 2000.

16. Guimarães SMR, Carvalho ACP, Guimarães JP, et al. Prevalência de alteração morfológica do processo estilóide em pacientes com desordem temporomandibular. Radiol Bras. 2006;39:407-11.

17. Milano V, Desiate A, Bellino R, et al. Magnetic resonance imaging of temporomandibular disorders: classification, prevalence and interpretation of disc displacement and deformation. Dentomaxillofac Radiol. 2000;29:352-61.

18. Barclay P, Hollender LG, Maravilla KR, et al. Comparison of clinical and magnetic resonance imaging diagnosis in patients with disk displacement in the temporomandibular joint. Oral Surg Oral Med Oral Pathol Oral Radiol Endod. 1999; 88:37-43.

19. Takaku S, Sano T, Yoshida M, et al. A comparison between magnetic resonance imaging and pathologic findings in patients with disc displacement. J Oral Maxillofac Surg. 1998;56:171-7.

20. Emshoff R, Innerhofer K, Rudisch A, et al. Clinical versus magnetic resonance imaging findings with internal derangement of the temporomandibular joint: an evaluation of anterior disc displacement without reduction. J Oral Maxillofac Surg. 2002;60:36-43.

21. Katzberg RW, Westesson PL, Tallents RH, et al. Anatomic disorders of the temporomandibular joint disc in asymptomatic subjects. J Oral Maxillofac Surg. 1996;54:147-55.

22. Kurita H, Ohtsuka A, Kobayashi H, et al. Is the morphology of the articular eminence of the temporomandibular joint a predisposing factor for disc displacement? Dentomaxillofac Radiol. 2000;29:159-62.

23. Augthun M, Müller-Leisse C, Bauer W, et al. Anterior disk displacement of the temporomandibular joint. Significance of clinical signs and symptoms in the diagnosis. J Orofac Orthop. 1998;59: 39-46.

24. Tenenbaum HC, Freeman BV, Psutka DJ, et al. Temporomandibular disorders: disc displacements. J Orofac Pain. 1999;13:285-90.

25. Toyama M, Kurita K, Koga K, et al. Magnetic resonance arthrography of the temporomandibular joint. J Oral Maxillofac Surg. 2000;58:97884.

26. Bell KA, Miller KD, Jones JP. Cine magnetic resonance imaging of the temporomandibular joint. Cranio. 1992;10:313-7.

27. Cholitgul W, Nishiyama H, Sasai T, et al. Clinical and magnetic resonance imaging findings in temporomandibular joint disc displacement. Dentomaxillofac Radiol. 1997;26:183-8. 